\title{
Whitehead Products and Projective Spaces
}

\author{
M. G. BARRATT, I. M. JAMES \& N. STEIN
}

Communicated by E. SPANIER

It has long been known how to express the homotopy groups of the classical projective spaces in terms of the homotopy groups of spheres. The object of this note is to provide expressions for the Whitehead products in similar terms. In particular, all Whitehead products are zero in the complex projective 3space, although this is not an $H$-space.

1. The Samelson product. Let $G$ be a topological group. Associated with a principal $G$-bundle over a space $X$ is a transgression operator

$$
\Delta: \pi_{r+1}(X) \rightarrow \pi_{r}(G), \quad r \geqq 0 .
$$

Let $p, q \geqq 1$ and let $[\xi, \eta] \varepsilon \pi_{p+q+1}(X)$ be the Whitehead product of $\xi \varepsilon \pi_{p+1}(X)$, $\eta \varepsilon \pi_{a+1}(X)$. We prove that

$$
(-1)^{p} \Delta[\xi, \eta]=\langle\Delta \xi, \Delta \eta\rangle,
$$

where the right hand side denotes the Samelson ${ }^{1}$ product, defined, as in $\$ 1$ of [15], by the use of commutators.

Instead of following [12] we prove (1.1) by a combination of results of Fox ([5]) and G. W. Whitehead ([15]). Let $Y^{A}$ denote the function space of basepoint-preserving maps of a complex $A$ into a space $Y$. We give $G^{A}$ the group structure inherited from $G$. From the given $G$-bundle $B$ over $X$ we obtain a $G^{A}$-bundle $B^{A}$ over $X^{A}$ and a corresponding transgression homomorphism

$$
\Delta^{A}: \pi_{1}\left(X^{A}\right) \rightarrow \pi_{0}\left(G^{A}\right) .
$$

When $A=S^{r}$, we can identify

$$
\pi_{1}\left(X^{A}\right)=\pi_{r+1}(X), \quad \pi_{0}\left(G^{A}\right)=\pi_{r}(G),
$$

so that $\Delta^{A}$ is transformed into $\Delta$. If $A=B \times C$, we embed $Y^{B}, Y^{C}$ in $Y^{A}$ in the usual way ([2]).

Take $A=S^{p} \times S^{\alpha}$; we can regard $S^{p+q}$ as obtained from $A$ by pinching

1 The first mention of such a product appears to be in [12]. 
$S^{p} \vee S^{a}$ to a point. The pinching map induces homomorphisms ${ }^{2} \theta, \varphi$ such that the following diagram is commutative:

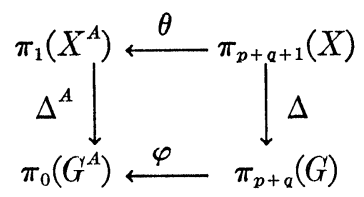

Let $\zeta \varepsilon \pi_{1}\left(X^{A}\right)$ be the commutator of the injections of $\xi \varepsilon \pi_{p+1}(X), \eta \varepsilon \pi_{q+1}(X)$ in $\pi_{1}\left(X^{A}\right)$, and let $\gamma \varepsilon \pi_{0}\left(G^{A}\right)$ be the commutator of the injections of $\Delta \xi, \Delta \eta$ in $\pi_{0}\left(G^{A}\right)$. Then $\Delta^{A} \zeta=\gamma$ since $\Delta^{A}$ is a homomorphism. In $\$ 8$ of [5] it is shown that $\zeta=(-1)^{p} \theta[\xi, \eta]$, and hence

$$
(-1)^{p} \phi \Delta[\xi, \eta]=\Delta^{A} \zeta=\gamma=\phi\langle\Delta \xi, \Delta \eta\rangle,
$$

by definition. Since $\phi$ is a monomorphism as is shown in [15], (1.1) follows.

2. The direct sum. We recall the construction of classifying spaces described by MILNor in [11]. Let $G$ be a topological group; MILNor makes $G$ act on $B_{m}$, the $(m+1)$-fold join of $G$ with itself, and denotes the orbit-space by $X_{m}$. Thus $B_{m}$ is a principal $G$-bundle over $X_{m}$; let $f_{m}: B_{m} \rightarrow X_{m}$ be the fibre map. Milnor embeds $B_{m}$ in a contractible subspace of $B_{m+1}$, so as to induce an embedding of $X_{m}$ in $X_{m+1}$. Now $X_{1}$ is the suspension of $G$, and the suspension homomorphism

$$
E: \pi_{r}(G) \rightarrow \pi_{r+1}\left(X_{1}\right)
$$

can be selected to be a right inverse of the transgression $\Delta$. Let

$$
E_{m}: \pi_{r}(G) \rightarrow \pi_{r+1}\left(X_{m}\right)
$$

denote the composition of $E_{1}=E$ and the injection induced by the inclusion $X_{1} \subset X_{m}$. The exact sequence of the fibration

$$
\cdots \rightarrow \pi_{r+1}\left(B_{m}\right) \stackrel{\rho_{m}}{\rightarrow} \pi_{r+1}\left(X_{m}\right) \stackrel{\Delta}{\rightarrow} \pi_{r}(G) \stackrel{\sigma_{m}}{\rightarrow} \pi_{r}\left(B_{m}\right) \rightarrow \cdots
$$

(where $\rho_{m}$ is induced by $f_{m}$, and $\sigma_{m}$ by the inclusion $G \subset B_{m}$ ) splits because $\Delta \circ E_{m}$ is the identity. This proves

Theorem (2.1). There is a direct sum decomposition

$$
\pi_{r}\left(X_{m}\right)=\rho_{m} \pi_{r}\left(B_{m}\right) \oplus E_{m} \pi_{r-1}(G), \quad r \geqq 2,
$$

where $\rho_{m}, E_{m}$ are monomorphisms. The injection of $\pi_{r}\left(X_{m}\right)$ in $\pi_{r}\left(X_{m+1}\right)$ annihilates the first summand and maps the second isomorphically by $E_{m+1} \circ E_{m}^{-1}$.

In view of (2.1) we distinguish three basic kinds of Whitehead product in the homotopy groups of $X_{m}$; thus, in $\pi_{p+q+1}\left(X_{m}\right)$ are

$$
\left.\begin{array}{ll}
\text { (i) } & {\left[\rho_{m} \xi, \rho_{m} \eta\right]=\rho_{m}[\xi, \eta]} \\
\text { (ii) } & {\left[E_{m} \alpha, E_{m} \beta\right]} \\
\text { (iii) } & {\left[\rho_{m} \xi, E_{m} \beta\right]}
\end{array}\right\} \begin{aligned}
& \xi \varepsilon \pi_{p+1}\left(B_{m}\right), \\
& \alpha \varepsilon \pi_{p}(G), \quad \eta \varepsilon \pi_{q+1}\left(B_{m}\right),
\end{aligned}
$$

2 These map additive groups into multiplicative groups. 
Any Whitehead product can be expressed as a linear combination of products of these three kinds.

The first kind are essentially Whitehead products in the homotopy groups of $B_{m}$, and there is nothing about these to discuss.

The second kind are related to the Samelson product in $G$, because

$$
\Delta_{m}\left[E_{m} \alpha, E_{m} \beta\right]=(-1)^{p}\langle\alpha, \beta\rangle
$$

by (1.1) and (2.1). When $m=1$ it is possible for $[E \alpha, E \beta]$ to have a component in the image of $\rho_{1}$ (as when $G=S^{3}$ ):

$$
[E \alpha, E \beta]=(-1)^{p} E\langle\alpha, \beta\rangle+\rho_{1}(\lambda) \text {. }
$$

However, when $m>1$ it follows from the latter part of (2.1) that this term is zero, i.e.

$$
\left[E_{m} \alpha, E_{m} \beta\right]=(-1)^{p} E_{m}\langle\alpha, \beta\rangle, \quad m \geqq 2 .
$$

Notice also that, by the definition of $E_{m}$,

$$
E_{m}(\alpha \circ \lambda)=E_{m} \alpha \circ E \lambda, \quad E_{m}(\beta \circ \mu)=E_{m} \beta \circ E_{\mu}
$$

where $\lambda \varepsilon \pi_{i}\left(S^{p}\right), \mu \varepsilon \pi_{i}\left(S^{q}\right)$; hence, by (3.59) of [14],

$$
\left[E_{m}(\alpha \circ \lambda), E_{m}(\beta \circ \mu)\right]=\left[E_{m} \alpha, E_{m} \beta\right] \circ(\lambda * \mu),
$$

where $(\lambda * \mu)=(-1)^{(i+1)(\alpha-j)} E^{\alpha+1} \lambda \circ E^{i+1} \mu$, by (2.4) of [3].

Finally, consider Whitehead products of the third kind. We say that

$$
\Delta\left[\rho_{m} \xi, E_{m} \beta\right]=0, \quad m \geqq 1 .
$$

For $\Delta$ can be factored through the injection of $\pi_{r}\left(X_{m}\right)$ in $\pi_{r}\left(X_{m+1}\right)$, which (by (2.1)) annihilates $\rho_{m} \xi$. Thus

$$
\left[\rho_{m} \xi, E_{m} \beta\right] \varepsilon \rho_{m} \pi_{p+a+1}\left(B_{m}\right) .
$$

The third kind of product may be regarded as a pairing of $\pi_{p+1}\left(B_{m}\right)$ with $\pi_{q}(G)$ to $\pi_{p+a+1}\left(B_{m}\right)$. We examine this in the classical cases when $G$ is a sphere.

3. The third kind of product in the classical case. Let $F$ denote the field of real numbers, complex numbers, or quaternions, and let $G \subset F$ be the (multiplicative) group of elements with unit norm. The classical projective spaces can be obtained by applying Mrlnor's construction to $G$, taking $B_{m}$ to be the unit sphere in the $(m+1)$-dimensional Euclidean space over $F$ and $X_{m}$ to be the corresponding projective space.

Let $q=1,2$ or 4 denote the dimension of $F$ over the reals, and let $n=q(m+1)$. We can make the identifications

$$
G=S^{a-1}, \quad X_{1}=S^{a}, \quad B_{m}=S^{n-1}
$$

in such a way that

$$
E_{1}=E: \pi_{r}(G) \rightarrow \pi_{r+1}\left(X_{1}\right)
$$


is the usual suspension homomorphism. Furthermore, we can regard $S^{n}$ as the result of pinching $X_{m} \subset X_{m+1}$ to a point; the pinching map will induce a homomorphism

$$
\mu_{m}: \pi_{r}\left(X_{m+1}\right) \rightarrow \pi_{r}\left(S^{n}\right) .
$$

Let $\beta_{m} \varepsilon E_{m} \pi_{q-1}\left(S^{\alpha-1}\right), \alpha_{m} \varepsilon \rho_{m} \pi_{n-1}\left(S^{n-1}\right)$ be the images of the classes of the identity maps $G \rightarrow G, B_{m} \rightarrow B_{m}$, respectively, so that

$$
\rho_{m}(\xi)=\alpha_{m} \circ \xi, \quad E_{m}(\lambda)=\beta_{m} \circ \lambda .
$$

We show how $\left[\rho_{m} \pi_{p+1}\left(B_{m}\right), E_{m} \pi_{r}(G)\right]$ can be determined from the value of $\left[\alpha_{m}, \beta_{m}\right]$, and we shall determine this element. (2.6) proves the first part of

Lemma 3.2 There is a $\gamma_{m} \varepsilon \pi_{n+q-2}\left(S^{n-1}\right)$ such that

$$
\left[\alpha_{m}, \beta_{m}\right]=\rho_{m}\left(\gamma_{m}\right) \text {. }
$$

Moreover, the orientations can be chosen so that

$$
E \gamma_{m}=\mu_{m}\left(\alpha_{m+1}\right) \text {. }
$$

This is the key lemma; $\mu_{m} \alpha_{m+1}$ will be determined later. Notice that $E$ is an isomorphism in (3.2).

First, take the real case, where $m=n-1$. The action of the fundamental group of $X_{m}$ is given by the antipodal transformation $T: S^{n-1} \rightarrow S^{n-1}$ of degree $(-1)^{n}$. Therefore

$$
\left[\alpha_{m}, \beta_{m}\right]=\left((-1)^{n}-1\right) \alpha_{m},
$$

and (3.2) follows at once.

Now let $F$ be the complex or quaternionic field. Take an $n$-element $E^{n}$ whose boundary is $S^{n-1}$ and a class $\lambda_{m} \varepsilon \pi_{n}\left(X_{m+1}, X_{m}\right)$ of a relative homeomorphism

$$
f_{m}^{\prime}:\left(E^{n}, S^{n-1}\right) \rightarrow\left(X_{m+1}, X_{m}\right)
$$

which coincides with $f_{m}$ on $S^{n-1}$. Thus $\lambda_{m}$ is a generator. Let $f_{m}, f_{m}^{\prime}$ induce homomorphisms $\rho_{m}, \rho_{m}^{\prime}$, respectively, in the commutative diagram

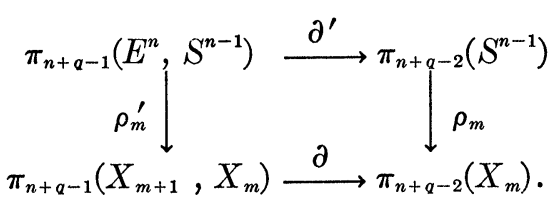

It follows from (2.1) and the exactness of the sequences of the pairs that the images of $\rho_{m}$ and $\partial$ coincide. Also $\rho_{m}$ is a monomorphism and $\partial^{\prime}$ is an isomorphism, while $\partial \circ \rho_{m}^{\prime}=\rho_{m} \circ \partial^{\prime}$. Therefore $\rho_{m}^{\prime}$ is a monomorphism, and $\partial$ maps its image isomorphically onto the image of $\partial$. By (1.4) of [9] the image of $\rho_{m}^{\prime}$ is a direct summand of $\pi_{n+a-1}\left(X_{m+1}, X_{m}\right)$, and the complementary summand is cyclic infinite, generated by the relative Whitehead product $\left[\lambda_{m}, \beta_{m}\right]$. Therefore the kernel of $\partial$ is freely generated by

$$
\left[\lambda_{m}, \beta_{m}\right]+\rho_{m}^{\prime} \tau_{m}
$$


for some $\tau_{m} \varepsilon \pi_{n+a-1}\left(E^{n}, S^{n-1}\right)$. The kernel of $\partial$ is, however, the image of the homomorphism

$$
j: \pi_{n+a-1}\left(X_{m+1}\right) \rightarrow \pi_{n+a-1}\left(X_{m+1}, X_{m}\right) .
$$

It follows from (2.1) that this image is generated by $j \alpha_{m+1}$; this proves

$$
\pm j \alpha_{m+1}=\left[\lambda_{m}, \beta_{m}\right]+\rho_{m}^{\prime} \tau_{m} .
$$

Since $\partial \circ j=0$, we deduce from this and (3.5) of [4] that

$$
\partial \rho_{m}^{\prime} \tau_{m}=\rho_{m} \partial^{\prime} \tau_{m}=\left[\alpha_{m}, \beta_{m}\right]
$$

hence $\gamma_{m}=\partial^{\prime} \tau_{m}$ in (3.2).

Now $\beta_{m}$, and hence $\left[\lambda_{m}, \beta_{m}\right]$, is annihilated by the homomorphism

$$
\mu_{m}^{\prime}: \pi_{n+q-1}\left(X_{m+1}, X_{m}\right) \rightarrow \pi_{n+q-1}\left(S^{n}\right)
$$

induced by pinching $X_{m}$ to a point. Since

$$
\mu_{m}=\mu_{m}^{\prime} \circ j,
$$

(3.3) implies

$$
\pm \mu_{m} \alpha_{m+1}=\mu_{m}^{\prime} \rho_{m}^{\prime} \tau_{m}= \pm E \partial^{\prime} \tau_{m}= \pm E \gamma_{m},
$$

as in $\$ 1$ of [6]. Hence $\mu_{m} \alpha_{m+1}= \pm E \gamma_{m}$, and we can choose orientations so as to obtain (3.2).

4. Conclusions. We can now carry out our intention of expressing Whitehead products in the classical projective spaces in terms of operations on the homotopy groups of spheres. We ignore products of the first kind since $B_{m}$ is itself a sphere, and $m=1$ since $X_{1}$ is itself a sphere.

Case 1. $F=$ real numbers. Here $B_{m}=S^{m}, \rho_{m}: \pi_{r}\left(S_{m}\right) \approx \pi_{r}\left(X_{m}\right)$ for $r \geqq 2$. If $T: S^{m} \rightarrow S^{m}$ is the antipodal map (of degree $(-1)^{m+1}$ ), the operation of $\beta_{m} \varepsilon \pi_{1}\left(X_{m}\right)$ on $\theta \varepsilon \pi_{r}\left(X_{m}\right)$ produces $\rho_{m} T_{*} \rho_{m}^{-1}(\theta)$. This determines products of the third kind:

$$
\begin{aligned}
& {\left[\rho_{m} \xi, \beta_{m}\right]=(-1)^{r}\left[\beta_{m}, \rho_{m} \xi\right] }=(-1)^{r}\left\{\rho_{m}(-\iota)^{m+1} \circ \xi-\rho_{m} \xi\right\} \\
&= \begin{cases}0 & m: \text { odd } \\
(-1)^{r}\left(-2 \rho_{m} \xi+\rho_{m}[\iota, \iota] \circ H_{0} \xi\right) & m: \text { even }\end{cases}
\end{aligned}
$$

(using Theorem 6.9 of [7]). Products of the second kind are trivial: $\left[\beta_{m}, \beta_{m}\right]=0$.

Case 2. $F=$ complex numbers. Here, again, products of the second kind are zero, for $G$ is abelian and the Samelson products vanish. Also $B_{m}=S^{2 m+1}$, an odd sphere.

To determine $\mu_{m} \alpha_{m+1}$ in (3.2), we examine the Steenrod squares in $X_{m+1}$. This shows that $\mu_{m} \alpha_{m+1}$ is trivial, and hence $\left[\alpha_{m}, \beta_{m}\right]=0$ if, and only if, $m$ is odd. When $m$ is odd, it follows from (7.4) of [1], or from (2.1) of [8], that all 
products of the third kind are zero. This can be proved directly from the standard representation of $X_{2 k+1}$ as a 2-sphere bundle over the quaternionic projective $k$-space. Notice, in particular, that $B_{3}=S^{7}$, and Whitehead products of all kinds are trivial in $X_{3}$, though it is not an $H$-space.

When $m$ is even, $\left[\alpha_{m}, \beta_{m}\right]=\alpha_{m} \circ \eta$, where $\eta \neq 0 \varepsilon \pi_{n}\left(S^{n-1}\right)$. Therefore

$$
\left[\alpha_{m}\left[\alpha_{m}, \beta_{m}\right]\right]=\alpha_{m} \circ[\iota, \iota] \circ \eta, \quad\left[\alpha_{m}\left[\alpha_{m}\left[\alpha_{m}, \beta_{m}\right]\right]\right]=0,
$$

and by (3.1) and (7.4) of [1]

$$
\begin{aligned}
{\left[\rho_{m} \xi, \beta_{m}\right]=\left[\alpha_{m} \circ \xi, \beta_{m}\right]=\alpha_{m} \circ \eta \circ E \xi } & +\alpha_{m} \circ[\iota, \iota] \circ \eta \circ E H_{0} \xi \\
& =\rho_{m}\left(\eta \circ E \xi+[\iota, \iota] \circ \eta \circ E H_{0} \xi\right) ;
\end{aligned}
$$

while $\left[\rho_{m} \xi, \beta_{m} \circ \nu\right]=0$ for any $\nu$ in any stem other than the 0 -stem.

Case 3. $F$ = quaternions. G. W. WhiteheAd has shown that if $\iota_{3}$ generates $\pi_{3}\left(S^{3}\right)$, then $\left\langle\iota_{3}, \iota_{3}\right\rangle$ generates ${ }^{3} \pi_{6}\left(S^{3}\right)$; the methods of $\S 9$ of [13] can be used. The products of the second kind are given by (2.3), (2.4).

By (2.10a) of [10], we have

$$
\mu_{m-1} \alpha_{m}= \pm m E^{4 m-4} \gamma,
$$

where $\gamma$ is the Hopf class in $\pi_{7}\left(S^{4}\right)$. Therefore

$$
\left[\alpha_{m}, \beta_{m}\right]= \pm(m+1) \rho_{m} E^{4 m-1} \gamma
$$

by (3.2), and the general product of the third kind can be deduced from this and (7.4) of [1] as before, though attention must be paid to the sign of the first term in this case.

The stable group of the 3-stem is cyclic of order 24, generated by the suspensions of $\gamma$. It follows that all products of the third kind are zero when $m \equiv-1$ $(\bmod 24)$.

The study of Whitehead products in the Cayley projective plane is much more awkward, since (2.1) breaks down.

\section{REFERENCES}

[1] W. D. Barcus \& M. G. Barratt, On the homotopy classification of extensions of a fixed map, Trans. Am. Math. Soc. 88 (1958), 57-74.

[2] M. G. Barratt, Track groups I, II, Proc. London Math. Soc. (3) 5 (1955), 71-106, 285-329.

[3] M. G. Barratt \& P. J. Hruton, On join operations in homotopy groups, Proc. London Math. Soc. (3) 3 (1953), 430-445.

[4] A. L. Blakers \& W. S. Massey, Products in homotopy theory, Ann. Math. 58 (1953), 295-324.

[5] R. H. Fox, On homotopy and torus homotopy groups, Ann. Math. 49 (1948), 471-510.

${ }^{3}$ In fact, $-\left\langle\iota_{3}, \iota_{3}\right\rangle$ is the Blakers-Massey element. 
[6] P. J. Hilton, Suspension theorems and the generalized Hopf invariant, Proc. London Math. Soc. (3), 1 (1951), 462-493.

[7] P. J. Hruton, On the homotopy groups of the union of spheres, J. London Math. Soc. 30 (1955), 154-172.

[8] P. J. Hilton \& J. H. C. Whitehead, Note on the Whitehead product, Ann. Math. 58 (1953), 429-442.

[9] I. M. JAmes, On the homotopy groups of certain pairs and triads, Quart. Journ. Math. Oxford (2) 5 (1954), 260-270.

[10] I. M. JAMES, On spaces associated with Stiefel Manifolds, Proc. London Math. Soc. (3) 9 (1959), 115-140.

[11] J. Milnor, Construction of universal bundles, II, Ann. Math. 63 (1956), 430-436.

[12] H. Samelson, A connection between the Whitehead and the Pontrjagin product, $\mathrm{Am}$. Journ. Math. 75 (1953), 744-752.

[13] H. Samelson, Groups and spaces of loops, Comm. Math. Helv. 28 (1954), 278-287.

[14] G. W. Whitehead, A generalization of the Hopf invariant, Ann. Math. 51 (1950), 192-237.

[15] G. W. Whitehead, On mappings into group-like spaces, Comm. Math. Helv. 28 (1954), $320-328$.

Brasenose College

and

Mathematical Institute

Oxford

and

Yale University

New Haven, Connecticut 